\title{
Working memory and situation model updating
}

\author{
GABRIEL A. RADVANSKY and DAVID E. COPELAND \\ University of Notre Dame, Notre Dame, Indiana
}

\begin{abstract}
Situation model updating requires managing the availability of information as a function of its relevance to the current situation. This is thought to involve some aspect of working memory. The present study assesses the relation between updating ability and various measures of working memory span or capacity. In addition, a primitive general measure of situation model processing, a situation model identification test, and its relation to updating ability was also assessed. The present experiment used a version of a paradigm developed by Glenberg, Meyer, and Lindem (1987) to assess updating. Although updating was observed in both anaphoric reading time and recognition test accuracy measures, the reading time measure was relatively weak. Importantly, the updating effect on the recognition test was unrelated to working memory capacity. In contrast, updating was related to performance on the situation model identification task. Specifically, people who were good at model processing were better able to keep associated objects available than were people who were less adept. There were no differences in the maintenance of dissociated objects. These results suggest that the relationship between situation model processing and working memory capacity is relatively weak.
\end{abstract}

Situation models (van Dijk \& Kintsch, 1983; Zwaan \& Radvansky, 1998), or mental models (Johnson-Laird, 1983), are complex mental representations that can simulate the situation described in a text. These representations capture important aspects of events in the world, such as space, time, causality, and so forth. Although we encounter event descriptions that are static, in most cases, the situations we read about change over time. For understanding to occur, we must update our models as new information is encountered about the unfolding of the events. The aim of this article is to assess the degree to which updating ability is affected by individual differences in working memory capacity.

Updating occurs by altering the structure of a situation model to capture the described changes. There are three general types of updating. The first is when new entities or properties are added to an existing model (e.g., Zwaan, Langston, \& Graesser, 1995) - for example, when a new character is introduced into the scene. The second is that the structural relations among entities in a model are altered to change their interrelations (e.g., Lutz \& Radvansky 1997)—-for example, when a character's goal goes from being unsatisfied to completed. The third type is when old entities or properties are removed from the model when no longer relevant (e.g., Glenberg, Meyer, \& Linden, 1987) - for example, when an object is in one loca-

This research was supported in part by a grant from the Army Research Institute, ARMY-DASW01-99-K-0001. We thank Andy Conway, Art Glenberg, and Michael Wenger for their helpful comments on earlier versions of this article. We also to thank Diane Berish, Anthony Bishara, Jackie Curiel, Lacey Nielson, Kris White, and Sarah Wieber for their assistance in collecting the data. Correspondence should be addressed to G. A. Radvansky, Department of Psychology, University of Notre Dame, Notre Dame, IN 46556 (e-mail: gradvans@nd.edu). tion, but a story character moves to another location, leaving the object behind. It is this third type that is the focus of the present study.

This type of updating, involving the removal or deemphasis of situational entities, has been demonstrated in a number of ways. In a study by Glenberg et al. (1987), people read a series of short passages. An example of one such passage is as follows:

1. Warren spent the afternoon shopping at the store.

2. He picked up/set down his bag and went over to look at some scarves.

3. He had been shopping all day.

4. He thought it was getting too heavy to carry.

In each passage a critical object (e.g., the bag) either was spatially associated with the protagonist (e.g., picked up) or was spatially dissociated from the protagonist (e.g., set down). If people are building models of the described situation, and they are updating these models during reading, then this manipulation should influence performance. People should perform better when the object had been associated than when it had been dissociated. The associated object stays in the foreground or focus of the model, and is more available. However, the dissociated object moves to the background, and is less available.

In one experiment, after reading a passage, people were presented with a probe word and had to indicate whether it was in the preceding passage. On some of the trials, this word was the critical object. Response times to these probes were slower when the object was dissociated than when it was associated with the protagonist. In another experiment, the last sentence of a passage contained an anaphoric "it" that referred to the critical object. The time to read these sentences was longer when the reference object was spatially dissociated than when it was associated. 
Situation model updating has also been studied in other paradigms as well. One method is to have people first memorize a map of a building, such as a research center containing many rooms. The location of several objects in those rooms is also learned. Afterward, people read a series of narratives that describe the movements of a protagonist through the memorized area. Knowledge about the objects in the building varies as a function of the protagonist's current (physical or mental) location in the building, with close objects being more available than far objects. This result is found using either memory probes (e.g., Morrow, Greenspan, \& Bower, 1987) or reading times for anaphoric sentences (e.g., Rinck \& Bower, 1995). (But see Wilson, Rinck, McNamara, Bower, \& Morrow, 1993, and Zwaan, Radvansky, Hilliard, \& Curiel, 1998, for limitations on these findings.) Thus people are updating spatial information about the situation as the protagonist moves about.

The updating of situation models has also been studied across a number of situation dimensions with a reading time analysis developed by Rolf Zwaan and his colleagues (e.g., Zwaan, Langston, \& Graesser, 1995; Zwaan, Magliano, \& Graesser, 1995). In this procedure, a text is coded for shifts along various situational dimensions, including time, space, causality, intentionality, and entity. For example, the introduction of a new entity would be marked as an entity shift. A move from one spatial location to another would be a spatial shift. Coded in this way, reading time data for individual clauses or sentences can be entered into a regression analysis. This process usually reveals that each of these dimensional shifts results in an increase in reading time, consistent with the idea that people are updating their situation models.

\section{Working Memory}

Situation models are complex representations. There is a lot of information that needs to be kept track of to keep the resulting model consistent and coherent, including information about the spatial-temporal framework, characteristics of situational entities, interrelations among those entities, and how and why events unfold over time. The better this information is managed, the more accurate and effective the situation models will be. The issue addressed here is whether there are individualdifferences in the ability to update situation models.

Because updating involves the active manipulation of information, it is a mental activity that falls under the rubric of working memory. One of the most studied aspects of working memory is its capacity - the amount of information that can be kept available while one is processing other types of information. A number of tests of working memory capacity have been developed and most relate to some aspect of language processing (see Daneman \& Merikle, 1996). Usually people are engaged in some task while temporarily retaining information, such as reading sentences or solving math problems. Essentially this creates a dual-task situation. In almost all cases, the focus is on the retention of knowledge over time, not the manipulation of and working with information, although there may be some validation of the secondary task (e.g., Turner \& Engle, 1989).

Working memory capacity may be related to situation model updating in that updating requires that a large amount of information be kept available to allow a person to shift from one situation to the next. Specifically, working memory must contain information about the relevant components of a situation, such as the spatial-temporal framework, the entities involved, their goals, motives, and properties, and the causal relations among events. When the model must be updated, new information must be kept available while it adds to or replaces old information. Some of the old information would need to be brought along to the new situation. The larger one's working memory capacity is, presumably, the more information can be brought into the new model of the described events.

Although capacity is a dimension along which people are known to vary and that likely influences situation model updating ability, it is not the only aspect of working memory that may be involved. An alternative idea is that updating ability may not reflect how large a person's working memory capacity is, but what they can do with what they have. Along these lines, one of the more recent ideas about working memory that has been advocated is the idea that the central executive works essentially as an attentional control mechanism (e.g., Baddeley, 1993; Engle, Tuholski, Laughlin, \& Conway, 1999; Kane \& Engle, 2000). Updating a model when an entity is removed from the situation suggests that attentional resources should be removed from an object that has moved from the foreground to the background.

Some working memory theorists have suggested that measures of working memory capacity reflect efficiency in the ability to suppress irrelevant information in the current stream of processing (e.g., Conway \& Engle, 1994; Rosen \& Engle, 1998). Thus, working memory may be involved in model updating by suppressing information that becomes irrelevant. When an object becomes dissociated from the protagonist, it is suppressed. It has been argued that suppression is a common mechanism used during comprehension. Irrelevant information is suppressed in the structures built during comprehension (e.g., Gernsbacher, 1990). People who have larger working memory spans, and are efficient at suppression, should be better at removing irrelevant information than people who have smaller working memory spans. Thus, people with higher working memory span scores should show a larger updating effect, and this should be due primarily to the processing of dissociated objects.

Different types of cognitive processes place different demands on the system. It may be that people's ability to update a model is related to how well they process situationspecific information in general (see Ericsson \& Kintsch, 1995; Glenberg, 1997). The exact nature of those processes is left ambiguous at this time. To gain a general measure 
of the ability to process event-specific knowledge, we developed a primitive measure that we call the situation identification test. This test is modeled after a procedure developed by Garnham (1981). In his study, people heard a series of sentences. Garnham found that people were more likely to misidentify a sentence as having been heard before if it described the same situation as the original sentence. People were much less likely to make errors when the sentences were altered in a similar fashion, but were likely to be interpreted as describing different situations. Thus, people were using situation models to make recognition decisions later (see also Bransford, Barclay, \& Franks, 1972; Rinck, Hähnel, \& Becker, 2001).

In our situation identification test, people read through a series of sentences. Then, after all of the sentences had been read, they were given a six-alternative forced-choice recognition test. The original sentence was never among the options, a fact that people were informed of. The task was to select the sentence that most closely described the same situation as the original. To perform this task, a person must have an understanding of what the previously described situation was, and then select the alternative that most closely matches that understanding. It should be noted that no situational updating is involved in the situation identification test. Thus, the relation between these two measures cannot be attributed to this component.

\section{Present Experiment}

In the present study, people were presented with the brief stories used by Glenberg et al. (1987). Each of these contained an anaphoric sentence that referred to an object earlier in the passage that had been spatially associated or dissociated from the protagonist. Reading times for these sentences were recorded. In addition, after each story, there were comprehension questions that asked about the anaphor in the last sentence. Accuracy rates to these questions were also recorded. This procedure is somewhat different from that in the Glenberg et al. study. That study used probes involving recall, whereas ours was more of a recognition test. We used both of our measures to assess whether people were updating their situation models.

In addition, people were also given a number of working memory tests. These included a word span test, the reading span test (Daneman \& Carpenter, 1980), the operation span test (Turner \& Engle, 1989), and the spatial span test (Shah \& Miyake, 1996). The reading span test was used because of its predictability in other studies of language comprehension(e.g., Daneman \& Merikle, 1996). The operation span test was used because it has been offered as a less language dependent measure of working memory capacity (e.g., Turner \& Engle, 1989). The spatial span test was included because the updating process assessed here is in some sense spatial, and performance on this test has been suggested as being correlated with spatial processing in situation models (e.g., Friedman \& Miyake, 2000). Finally, people were also given the situation identification test as a general measure of the ability to process and remember information about specific situations.

\section{METHOD}

\section{Subjects}

We tested 160 subjects in this experiment. These people were drawn from the subject pools at the University of Notre Dame and Indiana University South Bend and were reimbursed with partial class credit. All were native English speakers. The data from 2 of the subjects were discarded, one for having a situation identification test score at chance (4 out of 24), and the other one for not reading several of the stories.

\section{Materials and Procedure}

Memory span tests. There were four tests of working memory capacity. The most basic of these was a word span test. For this test, people were presented with a series of words in ever-increasing set sizes of 3-8, with three sets at each set size, and an additional three practice trials at set size 3 . The words were presented one at a time on a computer screen for $1 \mathrm{sec}$ each. At the end of each set, a series of question marks was displayed, one for each item in the set. The task was to recall the words in the order in which they were presented. People responded by typing their responses into the computer (although the experimenter typed them in for the other span tests). People typed "DK" ( $d$ on't $k$ now) for those items they could not recall. The order of the words was randomized for each subject.

For the reading span test, people were presented with a series of sentences in ever-increasing sets of $2-6$, with five sets at each set size, except for set size 6 , for which there were only three sets. The sentences were presented on a computer screen and the task was to read each sentence aloud. After each sentence, the experimenter advanced to the next sentence. Thus, in this and the other complex span tests, the experimenter controlled the rate of presentation. When a blank screen appeared, the task was to recall the final word from each of the sentences in each set. The experimenter typed these responses into the computer. Again, people were encouraged to recall the words in the order in which they were presented, and "DK" was typed for unknown responses. The order of the sentences was randomized for each subject.

For the operation span test, people were presented with a series of math problems followed by a word, such as " $(9 \times 1)+1=9$ " then "BOAT" in ever-increasing sets of $2-7$, with three sets at each set size, except for set size 2, for which there were six sets (the first three were considered practice). The problems and words were presented on a computer screen, and the task was to read each problem aloud, state and indicate whether the answer was correct or incorrect by pressing one of two buttons on a computer mouse, and then read the word aloud. When a blank screen appeared, the task was to recall the words from that set. The experimenter typed these responses into the computer. Again, people were encouraged to recall these in the order in which they were presented, and "DK" was typed for unknown responses. The order of the problems and words was randomized for each subject.

For the spatial span test, people were presented with a series of rotated letters (i.e., F, J, L, P, and R) in ever-increasing sets of 2-6, with five sets at each set size, except for only three at set size 6 . The letters were displayed on index cards, and the processing task was to state whether the letter was normal or mirror-imaged. The responses were recorded by the experimenter. After each set, a card was presented with eight locations mapped out around a circle at $45^{\circ}$ intervals $\left(0^{\circ}\right.$, or upright, was never used). The task was to indicate the position of the top of the letters in the current set in the order in which they were encountered. Note that the same location was never repeated within a set. The experimenter recorded these responses. Subjects were encouraged to guess if they were unsure.

Scoring of all of the span tests was done in a manner similar to that in Conway and Engle (1994). If a set was recalled correctly, then the score was equal to the number of words in that set. The scores for all of the correctly recalled sets were then totaled. 
Situation identification test. In this task, people were given a series of 24 sentences, which were drawn from or inspired by those materials used by Garnham (1981). ${ }^{1}$ These sentences were presented one at a time on a computer screen. During this initial presentation, the task was to rate the pleasantness of the situation described by each sentence. Responses were entered into the computer using a $1-7$ scale $(1=$ extremely unpleasant $;$ and $7=$ extremely pleasant $)$. Because this was only a cover task, these ratings were not recorded.

After all of the sentences were rated, people were given a surprise identification task. On each trial, six alternatives were presented that were variations of the original sentence. These six alternatives were presented in a random order on each trial for each person. The task was to select the item that most closely described the same situation as the sentence read and rated earlier. People were informed that the original sentences would never appear and to type their responses into the computer. No feedback was provided. There were three aspects of the original sentence that could have been altered. First, as in Garnham's (1981) study, was the prepositional phrase in the sentence. Second was the direct object of the sentence. Third was the verb in the sentence. The six alternatives were (1) altered prepositional phrase, (2) altered direct object, (3) altered verb, (4) altered prepositional phrase and direct object, (5) altered prepositional phrase and verb, and (6) altered direct object and verb. An example of six sentences for the original sentence The man lost a hand of poker at the card shark's are presented below. Sentence 2 is the correct response.

1. The man lost a hand of poker like the card shark.

2. The man lost some money at the card shark's.

3. The man won a hand of poker at the card shark's.

4. The man lost some money like the card shark.

5. The man won a hand of poker like the card shark.

6 . The man won some money at the card shark's.

Reading and memory task. The primary task in this study was based on that used in Glenberg et al.'s (1987) study. Subjects read five practice stories followed by a series of 24 experimental stories that were each four sentences long. There were also 24 filler stories that were three to five sentences long. For the experimental stories, the second sentence described an object becoming either spatially associated or spatially dissociated with the story protagonist. There were two versions of each story, and these versions were counterbalanced across subjects. The final sentence contained the word $i$ which referred to the critical object. These stories were presented on a computer, one sentence at a time, and reading times were recorded. People pressed the space bar with their left hand to advance to the next sentence. After the last sentence there was a probe question, such as "Did the word 'it' in the previous sentence refer to the bag?" Subjects were encouraged to respond as fast and as accurately as possible. Responses were made by pressing buttons on a computer mouse with the right hand. The left button was marked with a "Y" for "yes," and the right button was marked with an "N" for "no." The order of the experimental and filler stories was randomized for each person.

Experimental context. These measures were embedded in the context of a larger study. In addition to the tasks described earlier, also included were measures of processing speed, vocabulary, logical reasoning, a task that required reading relatively long passages, and a fan effect task. The results of these tasks will be considered elsewhere. A fixed order of tasks was set: (1) vocabulary, (2) speed, (3) situation identification, (4) word span, (5) reading span, (6) updating task, (7) spatial span, (8) long text comprehension and recognition, (9) operation span, (10) logical reasoning, and (11) fan effect. Testing was done across either two 1.5-h sessions or three 1-h sessions. If done over 2 days, Tasks 1-8 were done on Day 1 and Tasks 9-11 were done on Day 2. If testing was done over 3 days, Tasks 1-6 were done on Day 1, Tasks 7-9 on Day 2, and Tasks 10-11 on Day 3.

\section{RESULTS}

\section{Memory Span and Situation Identification}

Performance results for these tests are presented in Table 1. As can be seen in Table 2, the span tests were strongly correlated with one another. A decision was made to create a combined span score. This was done by dividing a person's score on each of these four tests by the maximum number possible, summing these proportions, and dividing by 4 . This combined span score is also presented in Table 1. Because the span scores were so interrelated, and the relation between each span test and the updating task was similar, only the combined scores are considered further. For those interested readers, the data for the individual span tests are presented in Appendix A and the regression analyses results in Appendix B.

Because this was the first use of the situation identification test, we will provide some additional psychometric characteristics. In our sample of 158, the scores ranged from 7 to 23 (out of 24) with a mean of 17.66, a median and mode of 18 , and a standard deviation of 3.3. As a measure of reliability, we computed Cronbach's alpha, which was .63. Thus, we found the situation identification test to be a reasonably reliable measure. The combined span test scores were not correlated with scores on the situation identification test $(r=.13, p=.12)$.

\section{Reading Times}

Overall, people read the final anaphoric sentence more slowly when it referred to a dissociated object $(234 \mathrm{msec} /$ syllable) than when it referred to an associated object $(229 \mathrm{msec} / \mathrm{syllable})\left[F(1,157)=5.72, M S_{\mathrm{e}}=387, p=.02\right]$. Although this effect is significant, it is rather small. Not surprisingly given the smallness of this reading time effect, it was unrelated to the individual difference measures.

\section{Recognition Accuracy}

Recognition errors were somewhat low. However, clear differences were observed. People made more errors to

Table 1

Summary of Mental Ability Test Scores

\begin{tabular}{lccccc}
\hline \multicolumn{1}{c}{ Measure } & $M$ & $S D$ & Minimum & Maximum & Scoring \\
\hline Word span & 35.0 & 12.9 & 9 & 77 & Count score \\
Reading span & 22.1 & 12.4 & 4 & 69 & Count score \\
Operation span & 21.6 & 11.1 & 0 & 54 & Count score \\
Spatial span & 28.6 & 19.0 & 0 & 84 & Count score \\
Combined span & .30 & .12 & .06 & .73 & Proportion correct \\
Situation identification & 17.7 & 3.3 & 7 & 23 & Number correct \\
\hline
\end{tabular}


Table 2

Correlations Among the Various Span Tests

\begin{tabular}{lccc}
\hline \multicolumn{1}{c}{ Measure } & Word & Reading & Operation \\
\hline Reading span & .58 & & \\
Operation span & .47 & .60 & \\
Spatial span & .40 & .54 & .39 \\
\hline
\end{tabular}

Note-All of these correlations were significant, at $p<.001$, with a Bonferroni correction.

the memory probes that referred to a dissociated object $(7.9 \%)$ than to an associated object $(4.7 \%)[F(1,157)=$ $\left.20.48, M S_{\mathrm{e}}=37, p<.001\right]$. Thus, when the object had been spatially associated in the described situation with the protagonist, information about it was more available than when it had been dissociated. This is consistent with previous research (Glenberg et al., 1987).

The individual difference comparisons are summarized in Table 3. As can be seen, working memory span did not appear to have a clear influence on performance on the updating data. In contrast, situation identification test scores were related to performance on the updating task. In general, the greater a person's situation identification score, the larger the updating effect. This was confirmed by a multiple regression analysis using the size of the associateddissociated difference as the dependent variable and the combined span and situation identification scores as the independent variables. For this analysis, composite working memory span was not a significant predictor $[\beta=-.06$, $t(155)=-0.75, p=.46]$, whereas situation identification was $[\beta=.15, t(155)=1.82, p=.07]$.

As can be seen in Table 3, where the data are broken down by quartiles to illustrate the pattern of performance, this is primarily due to people who scored higher on the situation identification test making fewer errors for the associated objects than the people who scored lower $[\beta=-.21$, $t(155)=-2.70, p=.008]$. However, there was no influence on errors made to the dissociated objects $[\beta=-.01$, $t<1$ ]. This is consistent with the idea that people who scored higher on the situation identification test were better able to maintain additional information in the focus of the situation model. Information that was removed from the focus was similarly less available for everyone.

\section{DISCUSSION}

This study replicated prior research in showing that information about objects that were associated with a story protagonist was more available than information for those that were dissociated (e.g., Glenberg et al., 1987). This was revealed in both the reading time and recognition data. For the reading time data, people read anaphoric sentences more slowly when the critical object was dissociated than when it was associated, although this effect was somewhat weak. This relatively weak reading time effect is likely due to the nature of the probe items, which was a recognition task for the present experiment but a recall task for the Glenberg et al. study.
Similarly, for the recognition data, more errors were made when the probed-for object was dissociated than when it was associated. This is consistent with the idea that when people read, information that remains as part of the situational foreground is more available than information that is moved out of focus and into the background. Shifts in spatial proximity are one way to accomplish this.

Interestingly, although it may be critically involved in many language processes (e.g., Daneman \& Merikle, 1996), working memory capacity was unrelated to the ability to update a situation model. However, performance on the situation identification test was related to updating ability. On the recognition task, although there was no difference for dissociated objects, for associated objects, people who were better at processing situation-specific knowledge made fewer errors than did people who scored lower.

\section{Implications for Situation Model Theory}

The results of the present experiment lend some insight into at least one way in which updating is achieved. For both the associated and dissociated versions of the passages, a new entity was introduced: the critical object. Thus, a new token was introduced into the model to represent that entity.

In the associated condition, that token had to be maintained as the spatial context shifted. With a shift in the spatial framework, the new model that results must have not only a token for the protagonist that moved to the new location, but also a token for the object that has in some way become a part of the protagonist. The reader needs to keep track of what situational components of the prior situation must be transferred to the new situation. Thus, the token for the critical object needs to be kept more active. Although more mental effort is required to keep the additional information active, at least in the present case, this appears to place a minimal drain on the system, so that no capacity limitations appear to be invoked. Instead, the critical factor is the facility with which people process situationspecific information (Ericsson \& Kintsch, 1995; Glenberg, 1997). This may involve such things as "knowing" what

Table 3

Individual Difference Comparisons of the Recognition Test Errors (in Percentages) Broken Down by Quartiles of the Composite Working Memory Span and Situation Identification Tests

\begin{tabular}{cccc}
\hline Measure & Associated & Dissociated & Difference \\
\hline
\end{tabular}

Working memory span

\begin{tabular}{llll} 
First $(0.16)$ & 4.4 & 6.9 & 2.5 \\
Second $(0.24)$ & 4.9 & 8.1 & 3.2 \\
Third (0.33) & 5.3 & 9.4 & 4.1 \\
Fourth (0.46) & 4.4 & 7.1 & 2.7 \\
Situation identification & & & \\
First (12.9) & 6.9 & 7.8 & 0.9 \\
Second (17.1) & 4.7 & 8.1 & 3.4 \\
Third (19.6) & 4.2 & 7.6 & 3.4 \\
Fourth $(21.8)$ & 3.0 & 7.8 & 4.8 \\
\hline
\end{tabular}

Note-Mean scores for the tests in each of the quartiles are given in parentheses. 
sorts of information should be brought along to the new situation as the context is altered.

In the dissociated condition, the token representing the critical object was backgrounded. There are two likely processes that can result in this outcome. One is a simple decay process. The situational component, the object token here, is no longer actively maintained, causing it to fall away from availability. The other alternative is an active suppression process. The situational component is removed from the present model by actively pushing it out. In the present study, there are a number of reasons to think that decay is operating.

It has been suggested that suppression is a common mechanism for removing newly irrelevant aspects of a mental model during comprehension (Gernsbacher, 1990). However, although there is evidence that suppression is operating at lower levels of processing (e.g., Gernsbacher, Varner, \& Faust, 1990), there currently exists no evidence to support the idea that suppression operates at the mental model level to update a situational shift. For example, in an explicit test of a suppression hypothesis with goal information, Lutz and Radvansky (1997; see also Radvansky \& Curiel, 1998) failed to find any evidence that no longer relevant goals are suppressed, even though they become markedly less available after they have been achieved. The evidence most consistent with the idea of suppression operating at the situation model level is when incorrect inferences have been drawn and a person's understanding of the described situation needs to be significantly reappraised (Hamm \& Hasher, 1992).

Moreover, if suppression were operating in this type of updating, then, as stated in the introduction, it would be reasonable to suspect that the working memory span tests would be predictive of performance. Specifically, it has been suggested that suppression ability is an integral component of what these working memory span tests are measuring (e.g., Conway \& Engle, 1994). However, no such relationship was observed.

Finally, previous research (e.g., Gernsbacher et al., 1990) suggests that if suppression were involved, a different pattern of results would have emerged. When suppression has been found to be involved in comprehension, good and poor comprehenders are equally effective at keeping relevant information active. Where they differ is in their ability to suppress irrelevant information. Good comprehenders quickly suppress this information, whereas poor comprehenders do not, leaving them with too much information to manage. Following this example, in the present study one would expect people who differ in updating ability to differ in the availability of dissociated objects, not associated objects. However, the opposite pattern was observed.

\section{Implications for Working Memory Theories}

The updating of situation models, by definition, must involve working memory. However, the most widely studied aspect of working memory, capacity, was found to be unrelated to this process. To understand how working memory is involved in model updating, one needs to consider the ways in which the information is being actively manipulated. That is, we must understand the procedures used by working memory. In terms of how working memory operates, this would seem to fall under the rubric of what has been termed the central executive (e.g., Baddeley, 1986). Thus, what we have found in this study is an influence of the central executive on cognition. However, the most extensively studied aspect of the central executive is its role as an attentional system (e.g., Baddeley, 1993; Kane \& Engle, 2000). There is little specification about how the central executive actively manipulates information. Thus, positing central executive involvement is not very informative.

In general, such a specification of central executive processes would involve a skill approach to working memory, where the handling of different types of information is better viewed as skills developed for different types of knowledge rather than simply the operation of general purpose components. Two recent approaches that incorporate such ideas are Ericsson and Kintsch's (1995) longterm working memory (LTWM) model and Glenberg's (1997) embodied cognition view.

According to the LTWM view (Ericsson \& Kintsch, 1995), some aspects of working memory operation can be viewed as skill performance. According to the LTWM framework, people can employ retrieval structures that are composed of a collection of retrieval cues. These retrieval structures can make specific types of knowledge in longterm memory more available. Thus, LTWM can be used to regulate the contents of working memory, and to make selected and relevant portions of information highly accessible. This is an aspect of cognition that is domain and skill dependent, not a general characteristic of a person. In the case of language comprehension, LTWM can be used by skilled readers to maintain those units of knowledge that would be relevant for understanding the current text.

For the present experiment, performance on our tasks reflects general processing skill at the situation model level. The ability to understand the reference of a text and to manipulate that information is something that clearly differs between people. Our situation identification test, although rudimentary, presumably taps into this ability. Moreover, although the updating task picked up on a different set of cognitive operations (altering one's mental representation of a described situation) relative to the situation identification task (matching alternative descriptors of a state of affairs), both of them presumably fall under the general rubric of situation-specific information processing skill. This is also why the working memory capacity tasks were not very predictive. They did not tap into this skill, but rather tapped into a different skill.

In Glenberg's (1997) view, memory and cognition are guided by mental representations and processes that take into account the nature of the human body and how it interacts with the world. Various working memory processes 
have developed to handle different types of interactions a person has with the world and the information contained in and derived from it. In the case of the present study, the knowledge that is relevant is how situations change when there is a shift in location. For example, it is important to know that a person can and is likely to carry certain objects with him/her when he/she moves from place to place, depending on the nature of the object and how the person is interacting with it. People who are better at understanding these events in the world can update their mental models more efficiently than can people whose cognitive skills are less developed.

What is needed to further understand these processes is an effort to better describe the different roles that working memory plays in the active manipulation of information beyond capacity or attentional control. Situation model updating processes should draw upon such procedures. Obviously, the present study does not offer any clear ideas about what such processes are. However, it does make it clear that these processes are in some sense unrelated to capacity (in any of the ways it has been characterized) but that there are individual differences in how working memory does its work.

\section{REFERENCES}

BAdDeley, A. D. (1986). Working memory. Oxford: Oxford University Press.

BADDELEY, A. D. (1993). Working memory or working attention? In A. D. Baddeley \& L. Weiskrantz (Eds.), Selection, awareness, and control: A tribute to Donald Broadbent (pp. 152-170). Oxford: Oxford University Press, Clarendon Press.

Bransford, J. D., Barclay, J., \& Franks, J. J. (1972). Sentence memory: A constructive versus interpretive approach. Cognitive Psychology, 3, 193-209.

Conway, A. R. A., \& ENgle, R. W. (1994). Working memory and retrieval: A resource-dependent inhibition model. Journal of Experimental Psychology: General, 123, 354-373.

DANEMAN, M., \& CARPENTER, P. (1980). Individual differences in working memory and reading. Journal of Verbal Learning \& Verbal Behavior, 19, 450-466.

Daneman, M., \& Merikle, P. M. (1996). Working memory and language comprehension: A meta-analysis. Psychonomic Bulletin \& Review, 3, 422-433.

Engle, R. W., Tuholski, S. W., Laughlin, J. E., \& Conway, A. R. A. (1999). Working memory, short-term memory and general fluid intelligence: A latent variable approach. Journal of Experimental Psychology: General, 128, 309-331.

Ericsson, K. A., \& Kintsch, W. (1995). Long-term working memory. Psychological Review, 102, 211-245.

Friedman, N. P., \& MiYAKe, A. (2000). Differential roles for visuospatial and verbal working memory in situation model construction. Journal of Experimental Psychology: General, 129, 61-83.

Garnham, A. (1981). Mental models as representations of text. Memory \& Cognition, $9,560-565$.

Gernsbacher, M. A. (1990). Language comprehension as structure building. Hillsdale, NJ: Erlbaum.

Gernsbacher, M. A., VArner K. R., \& FAust, M. E. (1990). Investi- gating differences in general comprehension skill. Journal of Experimental Psychology: Learning, Memory, \& Cognition, 16, 430-445.

GLENBERG, A. M. (1997). What is memory for? Behavioral \& Brain Sciences, 20, 1-55.

Glenberg, A. M., Meyer, M., \& Lindem, K. (1987). Mental models contribute to foregrounding during text comprehension. Journal of Memory \& Language, 26, 69-83.

HAMм, V. P., \& HASHER, L. (1992). Age and the availability of inference. Psychology \& Aging, 7, 56-64.

JOHNSON-LAIRD, P. N. (1983). Mental models: Towards a cognitive science of language, inference and consciousness. Cambridge, MA: Harvard University Press.

KANE, M. J., \& ENGLE, R. W. (2000). Working-memory capacity, proactive interference, and divided attention: Limits on long-term memory retrieval. Journal of Experimental Psychology: Learning, Memory, \& Cognition, 26, 336-358.

Lutz, M. F., \& Radvansky, G. A. (1997). The fate of completed goal information in narrative comprehension. Journal of Memory \& Language, 36, 293-310.

Morrow, D. G., Greenspan, S. L., \& Bower, G. H. (1987). Accessibility and situation models in narrative comprehension. Journal of Memory \& Language, 26, 165-187.

Radvansky, G. A., \& Curiel, J. M. (1998). Narrative comprehension and aging: The fate of completed goal information. Psychology \& Aging, 13, 69-79.

Rinck, M., \& Bower, G. H. (1995). Anaphor resolution and the focus of attention in situation models. Journal of Memory \& Language, 34, 110-131.

Rinck, M., HÄHNel, A., \& Becker, G. (2001). Using temporal information to construct, update, and retrieve situation models of narratives. Journal of Experimental Psychology: Learning, Memory, \& Cognition, 27, 67-80.

Rosen, V. M., \& ENGLE, R. W. (1998). Working memory capacity and suppression. Journal of Memory \& Language, 39, 418-436.

Shah, P., \& MiYake, A. (1996). The separability of working memory resources for spatial thinking and language processing: An individua differences approach. Journal of Experimental Psychology: General, 125, 4-27.

Turner, M. L., \& Engle, R. W. (1989). Is working memory task dependent? Journal of Memory \& Language, 28, 127-154.

van DiJK, T. A., \& KInTSCH, W. (1983). Strategies in discourse comprehension. New York: Academic Press.

Wilson, S. G., Rinck, M., McNamara, T. P., Bower, G. H., \& MorROW, D. G. (1993). Mental models and narrative comprehension: Some qualifications. Journal of Memory \& Language, 32, 141-154.

ZwaAn, R. A., Langston, M. C., \& Graesser, A. C. (1995). The construction of situation models in narrative comprehension: An eventindexing model. Psychological Science, 6, 292-297.

Zwaan, R. A., Magliano, J. P., \& Graesser, A. C. (1995). Dimensions of situation model construction in narrative comprehension. Journal of Experimental Psychology: Learning, Memory, \& Cognition, 21, 386-397.

ZwaAn, R. A., \& RAdVAnsky, G. A. (1998). Situation models in language comprehension and memory. Psychological Bulletin, 123, 162-185.

Zwaan, R. A., Radvansky, G. A., Hilliard, A. E., \& Curiel, J. M. (1998). Constructing multidimensional situation models during reading. Scientific Studies of Reading, 2, 199-220.

\section{NOTE}

1. For interested readers, a Web page dedicated to the situation identification test can be found at http://www.nd.edu/ memory/situationidentification. 
APPENDIX A

Recognition Errors (in Percentages) Broken Down Into Quartiles (Mean Test Scores in Each Quartile Presented in Parentheses)

\begin{tabular}{lccc}
\hline \multicolumn{1}{c}{ Measure } & Associated & Dissociated & Difference \\
\hline Word span & & & \\
First (22.2) & 5.7 & 8.2 & 2.5 \\
Second (30.4) & 4.4 & 6.9 & 2.5 \\
Third (37.4) & 3.3 & 8.1 & 4.8 \\
Fourth (53.1) & 5.1 & 7.9 & 2.8 \\
Reading span & & & \\
First (9.5) & 3.4 & 6.0 & 2.6 \\
Second (16.8) & 6.3 & 7.9 & 1.6 \\
Third (23.0) & 5.5 & 9.9 & 4.4 \\
Fourth (39.1) & 3.7 & 7.7 & 4.0 \\
Operation span & & & \\
First (8.3) & 4.8 & 9.4 & 4.6 \\
Second (16.9) & 5.6 & 6.0 & 0.4 \\
Third (23.9) & 4.3 & 9.1 & 4.8 \\
Fourth (36.8) & 4.3 & 6.8 & 2.5 \\
Spatial span & & & \\
First (7.3) & 3.9 & 6.0 & 2.1 \\
Second (19.9) & 5.5 & 9.0 & 3.5 \\
Third (33.2) & 4.0 & 8.9 & 4.9 \\
Fourth (55.5) & 5.9 & 7.7 & 1.8 \\
\hline
\end{tabular}

APPENDIX B

Multiple Regression Analyses for the Individual Span Tests

\begin{tabular}{lrrr}
\hline \multicolumn{1}{c}{ Measure } & \multicolumn{1}{c}{$t$} & \multicolumn{1}{c}{$t$} & $p$ \\
\hline Word span & -.05 & -0.60 & .55 \\
Situation identification & .14 & 1.76 & .08 \\
Reading span & .01 & 0.12 & .91 \\
Situation identification & .14 & 1.71 & .09 \\
Operation span & -.06 & -0.72 & .47 \\
Situation identification & .14 & 1.78 & .08 \\
Spatial span & -.08 & -0.95 & .34 \\
Situation identification & .15 & 1.85 & .07 \\
\hline
\end{tabular}

(Manuscript received March 13, 2001;

revision accepted for publication August 7, 2001.) 